\title{
Sintomatología y resiliencia ante el acoso relacional en México
}

\author{
Symptomatology and resilience \\ during relational harassment in México
}

\author{
Alejandra Elizabeth Pérez Pérez, Berenice Jiménez Rodríguez \\ y Rozzana Sánchez Aragón ${ }^{I}$
}

\begin{abstract}
RESUMEN
Experimentar acoso relacional, referido a conceptos tales como hostigamiento, obsesión o violación psicológica, tiene importantes efectos en diferentes aspectos de la vida de las víctimas, por lo que esta investigación tuvo como objetivos validar dos escalas para medir los síntomas y la resiliencia ante dicho acoso, y examinar asimismo las diferencias por sexo y por la relación que la víctima tiene con el acosador. Los resultados indican la obtención de medidas válidas y confiables para ambas variables. Por un lado, se agrupan ciertos síntomas psicológicos (emocionales, de ansiedad, mentales, sociales y espirituales), y por otro los síntomas en el estilo de vida (económicos, conductuales y autodestructivos). En el caso de la resiliencia, esta fue unidimensional. En cuanto a diferencias encontradas, las mujeres manifestaron una mayor intensidad en algunos de sus síntomas, así como en la resiliencia espiritual. También se observó que los efectos del acoso se experimentan más vivamente entre más cercana es la relación con el acosador. Así, resulta evidente la importancia de la contribución metodológica de esta investigación, basada en el desarrollo de medidas válidas, y el conocimiento teórico obtenido de la experiencia del acoso para así mejorar la orientación y el tratamiento al respecto.
\end{abstract}

Palabras clave: Víctimas; Acosador; Relaciones personales; Medición.

\begin{abstract}
Experiencing relational harassment, through stalking, harassment, obsession, or psychological offense, has substantial effects on the lifestyle of victims. The present study aimed at validating two scales to measure symptoms and resilience to relational harassment, and to examine the differences by sex and type of relationship victims had with their stalker. Results indicate valid and reliable measures for both variables. On the one hand, psychological (emotional, anxiety, mental, social and spiritual) symptoms and other lifestyle (economic, behavioral and self-destructive) symptoms grouped together, while resilience showed a one-dimensional nature. Women expressed greater intensity in some of their symptoms but also higher spiritual resilience. Harassment effects are more intensely experienced the closer the victims'relationship with the stalker. The methodological contribution of the present study resides in the development of a valid measure, and the theoretical knowledge obtained from harassment experience in order to improve both preventive advice and treatment.
\end{abstract}

Key words: Victims; Stalkers; Personal relationships; Measurement.

${ }^{1}$ Facultad de Psicología, Universidad Nacional Autónoma de México, Av. Universidad 3004, Col. Copilco Universidad, Del. Coyoacán, 04510 Ciudad de México, México, correo electrónico: rozzara@unam.mx. Artículo recibido el 22 de mayo y aceptado el 28 de agosto de 2017. 
$\mathrm{L}$ a búsqueda insistente que lleva a cabo un individuo que pretende establecer una relación íntima (física o simbólica de compañerismo, amistad, romance o cercanía) con otra persona, a pesar de que esta no la desea, conlleva que los involucrados estén en una situación disyuntiva y disfuncional que se conoce como intrusión relacional obsesiva (IRO en lo sucesivo) (Spitzberg y Cupach, 2014).

Este tipo de persecución, también conocida como "acoso", probablemente ha existido siempre en las sociedades debido a la construcción cultural de lo que es y lo que implica el amor romántico y apasionado (Meloy, 1999; Meloy, Hoffmann, Guldimann y James, 2012). Por ejemplo, la poesía, el canto o la narración histórica sugieren que la obsesión motivada por un objeto de amor está llena de "pasiones paradójicas, confusas y contrarias, como el odio, los celos y el egoísmo, la atracción y la repulsión, la intimidad y el miedo [...] el deseo de aceptación y la hostilidad" (Kamir, 2001, p. 16). Actualmente, la IRO se ubica dentro de los diferentes tipos de agresión íntima (abuso de la pareja, violación, acoso sexual, hostigamiento (bullying) y hostigamiento laboral (mobbing), ya que genera en los receptores de tales actos altos niveles de emociones y sentimientos negativos, como miedo, ansiedad y sentimientos de victimización (Sinclair, 2012).

La literatura sugiere que las conductas de acecho surgen a partir de los restos de una relación incipiente o ya desarrollada (Duntley y Buss, 2012; Miller, 2012; Nicastro, Cousins, y Spitzberg, 2000), por lo que el acoso no solo se ha considerado como una ofensa, sino como un crimen que se caracteriza por su carácter repetitivo, persistente y repentino (Mullen, Pathé y Purcell, 2001; Spitzberg y Cupach, 2007).

A pesar de la controversia sobre los límites precisos de las conductas de acoso, hay consenso en cuanto a que tales comportamientos pueden incluir una estrecha vigilancia, el acoso telefónico o por correo electrónico, la usurpación de la identidad de la víctima, las amenazas, las agresiones físicas o sexuales, e incluso el intento de asesinato (McEwan, Pathé y Ogloff, 2011; Miller, 2012; Spitzberg y Cupach, 2014). Sin embargo, debido a que el acoso se compone también de actos que pueden parecer benignos o no representar una amenaza inmediata contra la integridad de la persona, como regalar cosas o mostrar interés por sus actividades sociales, el acoso no es denunciado por las víctimas, lo que representa un problema para que los sistemas de justicia determinen su prevalencia (Breiding et al., 2014). Datos estadísticos de esa prevalencia en Estados Unidos indican que este fenómeno afecta a 7.5 millones de personas anualmente, a $8 \%$ entre las mujeres y a $2 \%$ entre los hombres, con edades de 18 a 29 años; asimismo, se ha encontrado que la gran mayoría de los acosadores son hombres ( $87 \%$ ); y que en el caso de $59 \%$ de las mujeres y $30 \%$ de los hombres víctimas, el perpetrador es o ha sido su ex pareja (Baum, Catalano, Rand y Rose, 2009; Breiding et al., 2014; Langhinrichsen-Rohling, 2012; Tjaden y Thoennes, 2000). En México, las cifras existentes se reducen a la experiencia de las mujeres. De hecho, 63\% de mujeres de entre 15 y 49 años de edad han enfrentado situaciones de violencia sexual, tales como abuso, intimidación, acoso u hostigamiento por parte de su pareja o de otras personas (Casique, 2017).

La IRO tiene efectos potencialmente devastadores sobre las víctimas debido a que los perpetradores con frecuencia acosan a sus víctimas durante años, siendo el promedio de tiempo de cuatro a dieciocho meses (Spitzberg y Cupach, 2014). Durante ese tiempo, el perseguidor puede abrumar psicológicamente al objeto de su afecto a través de incesantes llamadas a todas horas, de notas y regalos no deseados que aparecen en lugares sorprendentes; también apareciéndose en el trabajo, la escuela, los lugares de recreación y socialización que frecuenta la víctima, e inclusive en su propia casa (Spitzberg, Cupach y Ciceraro, 2010). Además, los acosadores buscan maneras ingeniosas de involucrarse en la vida de la víctima, como infiltrarse entre sus amistades y familia, uniéndose a grupos de recreación a la que pertenece la víctima o realizando conductas cada vez más riesgosas e intimidatorias, tales como proferir amenazas de allanamiento, secuestro, restricción física o sexual, o de daño a la víctima o a sus seres queridos si la intimidad no es correspondida, o bien dejar objetos amenazantes o imágenes que desprestigian a la víctima en espacios públicos, entre otros (Duntley y Buss, 2012; Spitzberg y Cupach, 2014). 
Cuando se vive una situación de acoso, la víctima experimenta momentos estresantes que debilitan los sistemas de protección normales que proveen a las personas una sensación de control y significado, lo que ocasiona cambios en sus respuestas fisiológicas, emociones, cognición y memoria (Spitzberg y Cupach, 2014). Así, casi todas las víctimas sufren efectos psicológicos nocivos típicamente conceptualizados como síntomas muy variados en su tipo, intensidad e implicaciones en el corto y largo plazo (Davis, Coker y Sanderson, 2002; Sheridan y Lyndon, 2012). La mayoría de los estudios (Bjerregaard 2000; Brewster, 1999; Kamphuis, Emmelkamp y Bartak, 2003; Kuehner, Gass y Dressing, 2007; Nicastro et al., 2000; Sheridan y Lyndon, 2012) señalan que el acoso aumenta el miedo, la desconfianza hacia otros, provoca náuseas persistentes y dolores de cabeza, exacerbación de problemas médicos preexistentes, uso y abuso de sustancias, confusión, nerviosismo, ansiedad, problemas gástricos, alteraciones crónicas del sueño, debilidad o cansancio persistente y alteraciones del apetito.

Además, muchas de las víctimas sufren también pérdidas económicas y sociales, pues tienen que cambiar constantemente su número de teléfono, dirección, empleo, escuela o aficiones, invertir en tecnología para su protección (alarmas, objetos de seguridad, etc.) y servicios (guardaespaldas), y restringir sus actividades sociales y vida pública (Mohandie, Meloy, McGowan y Williams, 2006; Spitzberg y Cupach, 2007). Por si no bastara lo anterior, las víctimas informan aún más síntomas cuando los comportamientos de acoso son más penetrantes, intensos y persistentes (Blaauw, Winkel, Arensman, Sheridan y Freeve, 2002).

Hay que señalar en este punto que la aproximación psicológica tradicional desde la que se ha abordado la IRO se ha centrado en los efectos negativos de esta en la salud del individuo, los que generan el trastorno de estrés postraumático (a raíz de la persecución) y su consiguiente sintomatología (Bonanno, 2004). Sin embargo, desde otra perspectiva se encuentra la idea de que la mayor parte de las personas se encuentran expuestas al menos un evento desafortunado o amenazante en su vida (Folkman, 2013; Ozer, Best, Lipsey y Weiss, 2003), que no obstante acentúa la capacidad que tienen las mismas de soportar notablemente bien la conmoción, sin alteraciones aparentes en su desempeño en el trabajo o en las relaciones íntimas, y que parecen vencer los nuevos retos con aparente facilidad (Bonanno, 2004; Bonanno, Westphal y Mancini, 2011). A este fenómeno se le conoce como resiliencia.

Con base en estos dos enfoques respecto a las formas de reaccionar ante la IRO, Spitzberg y Cupach (2014) proponen que los efectos de dicho problema cubran dos dimensiones básicas de la vida del individuo: 1) las consecuencias de orden relacional, que se refieren a las irrupciones que la persona y miembros cercanos a ella experimentan, por lo que los autores han desarrollado ocho dimensiones de síntomas emergentes frente a la experiencia de acoso relacional; a saber, alteraciones de conducta, trastornos económicos, mentales, emocionales, de ansiedad, sociales, espirituales y autodestructivos, y 2) las de orden personal, que atañen básicamente a la manifestación de la resiliencia, que representa una actitud positiva ante la adversidad y que se compone de la percepción de que la vida puede ser "mejorable", el reconocimiento y la valoración de la red social y su función de soporte (Spitzberg y Cupach, 2001). Por tanto, la resiliencia incluye: resistir el suceso y rehacerse a sí mismo, lo que es factible gracias a que, como rasgo de personalidad, genera respuestas que proveen de una visión del yo positiva al poder aprender de la desgracia, las que evidencian los recursos que se poseen para lidiar con las amenazas; lo que trae consigo que el individuo se sienta seguro de sí mismo, encuentre que las experiencias negativas tienen un propósito significativo en la vida, confíe en su capacidad de influir en lo que le sucede y crea que se puede aprender de las experiencias positivas y de las negativas (Bonanno et al., 2011; Tugade y Fredrickson, 2004).

De acuerdo con lo planteado hasta ahora respecto al fenómeno de la IRO, se puede concluir que, al ser un evento traumático que implica amenaza, trae consigo efectos negativos conocidos también como síntomas, y puede desencadenar efectos positivos, como la expresión de la resiliencia.

El hecho de que la mayor parte de la investigación realizada al respecto haya sido en población anglosajona, y de que su prevalencia sea mundial, su estudio en nuestra cultura se vuelve una necesi- 
dad imperante. Por ello, los objetivos del presente trabajo fueron los de validar la Escala de Síntomas y la Escala de Resiliencia ante el acoso relacional en la víctima, y examinar las posibles diferencias respecto a los síntomas y la resiliencia en función del sexo y la relación con el acosador.

\section{MÉTODO}

\section{Participantes}

Se conformó una muestra no probabilística accidental (Kerlinger y Lee, 2002) de 717 participantes en total, todos ellos residentes en la Ciudad de México, de los cuales 504 fueron mujeres y 213 hombres, cuyas edades fueron de 16 a 65 años $(\mathrm{M}=23.13$ años $)$. La distribución del nivel educativo fue como sigue: $29 \%$ de bachillerato, $60 \%$ de licenciatura y $11 \%$ que no especificó su escolaridad, pero cuyas respuestas fueron tomadas en cuenta para el análisis. Para participar fue necesario que las personas respondieran a afirmativamente a la pregunta " $¿ H a$ sido acosado(a) en su vida?". Al preguntárseles respecto a su relación con el acosador, 131 respondieron que era un extraño, 159 un conocido, 129 un colega, 98 un amigo(a), 24 un familiar, 34 una cita casual, 38 una cita seria, 19 el cónyuge, 58 una ex pareja y 23 otro tipo de relación.

\section{Instrumentos}

Las Escalas de Síntomas ante el Acoso y de Resiliencia ante el Acoso forman parte del Autorreporte de Victimización, adaptado de la Encuesta de Intrusión Relacional Obsesiva de Spitzberg y Cupach (2014), que en su primera sección incluye preguntas sobre los datos sociodemográficos de cada participante y especificidades de la situación de acoso que experimentaron; en la segunda sección se presentan las medidas de síntomas y resiliencia, teniendo ambas escalas como estímulo la pregunta "¿En qué medida usted experimentó cualquiera de las siguientes situaciones, como resultado directo de la persecución no deseada de esta persona?".

\section{Escala de Síntomas ante el Acoso}

Está compuesta por un total de ocho agrupaciones de reactivos que miden síntomas 1) conductuales, es decir cambios en la rutina diaria; 2) económicos, referentes a la pérdida de ingresos, trabajo, gastos en seguridad o acciones legales; 3) mentales, como dificultad para recordar y falta de concentración; 4) emocionales, referentes a la tristeza y depresión; 5) de ansiedad, como tensión, pánico o paranoia; 6) sociales, que aluden a problemas de interacción con sus relaciones cercanas; 7) espirituales, es decir pérdida de la confianza en las instituciones y relaciones de la víctima, y 8) autodestructivos, son los intentos o pensamientos suicidas.

\section{Escala de Resiliencia ante el Acoso}

Consta de seis grupos de reacciones resilientes: 1) personales, que implican la capacidad de recuperarse del evento, aceptar el cambio y hacer frente a la adversidad; 2) de mejora, que aluden a una mejor relación con el perpetuador del acoso; 3 ) sociales, referentes a la percepción positiva de la red social; 4) de apertura, esto es, las competencias que se descubrieron o desarrollaron para enfrentar y sobreponerse a la experiencia; 5) De afrontamiento, o sea, el esfuerzo del individuo (recursos conductuales) que revelan una capacidad superior de la que la persona creía tener para enfrentar el incidente, y 6) espirituales, referidas a las sensaciones de apreciación por la vida y una visión optimista del futuro.

El formato de respuesta para ambas escalas es de tipo Likert con seis opciones, con recorrido de 1 (Nunca) a 6 (Más de diez veces), cuya media teórica es 3.5.

\section{Procedimiento}

Para la aplicación del instrumento se acudió a lugares públicos (escuelas, parques, centros comerciales) en donde se preguntaba a las personas si eran o habían sido víctimas de acoso relacional (brindándoles la definición conceptual e incluso operacional); cuando la respuesta era afirmativa, se les solicitaba contestar una serie de preguntas relativas a ese problema. A cada uno de los participantes se le aseguró la confidencialidad de sus 
respuestas y de que en caso de desear suspender su participación, podrían hacerlo libremente; asimismo, se resolvieron las dudas que surgieron ante las pruebas y se les indicó que los datos proporcionados se tratarían únicamente con fines de investigación.

\section{RESULTADOS}

Para cumplir con el objetivo de la validación de ambas escalas en una muestra mexicana, primero se realizó el proceso de traducción-retraducción de los reactivos originales de ambos instrumentos; después, tras la captura de las respuestas dadas por los participantes a las escalas, se siguió el procedimiento de validación de pruebas psicológicas culturalmente sensibles propuesto por Reyes y García
(2008) y que se fundamenta en los planteamientos de Cronbach y Meehl (1955), para lo cual se calcularon estadísticos descriptivos (frecuencia y medidas de tendencia central), pruebas $t$ de Student para discriminar cada reactivo. Debe decirse que no se eliminaron reactivos en la Escala de Síntomas, y de la Escala de Resiliencia, solo dos. Se llevó a cabo un análisis de correlación producto-momento de Pearson entre los reactivos para identificar el tipo de rotación a solicitar en el análisis factorial, que en ambos casos fue ortogonal (tipo varimax). Respecto a este último análisis de la escala de síntomas se obtuvo un índice KMO de .914; el valor de la Prueba de Esfericidad de Bartlett fue de $2667.053(\mathrm{gl}=28, p=.000)$ y se identificaron dos factores con valores propios superiores a 1 que explicaron $66.50 \%$ de la varianza (Tabla 1).

Tabla 1. Análisis factorial de la Escala de Síntomas por reactivo.

\begin{tabular}{|c|c|}
\hline FACTORES & $\begin{array}{c}\text { Carga } \\
\text { factorial }\end{array}$ \\
\hline Factor 1. Síntomas psicológicos & $\alpha=.896$ \\
\hline $\begin{array}{l}\text { Síntomas de ansiedad (e.g. ataques de pánico, tensión, sentimiento de paranoia, miedo general a los demás, timi- } \\
\text { dez, pesadillas, desconfianza en los demás, etc.) }\end{array}$ & .806 \\
\hline $\begin{array}{l}\text { Síntomas mentales (e.g. dificultad para recordar cosas o para concentrarse, dificultad para tomar decisiones, tener } \\
\text { la mente "en blanco", pensar cosas desagradables o criticar a los demás, estado de alerta a su alrededor, no entender } \\
\text { lo que otros dicen, ser optimista sobre cualquier cosa, percepción de sí mismo como un objeto, culparse a sí mismo } \\
\text { por cosas, obsesionarse con las cosas, etc.) }\end{array}$ & .804 \\
\hline $\begin{array}{l}\text { Síntomas sociales (e.g. ser demasiado crítico con los demás, ponerse por debajo de los demás, arrebatos de ira } \\
\text { injustificada, reaccionar exageradamente a lo que otros dicen o hacen, celos injustificados, posesividad hacia otros, } \\
\text { alejamiento de los demás, evitación de situaciones sociales, etc.) }\end{array}$ & .749 \\
\hline \multicolumn{2}{|l|}{ Varianza explicada $=\mathbf{3 6 . 7 9} \%$ Media $=\mathbf{2 . 2 5} \mathrm{DS}=\mathbf{1 . 2 7}$} \\
\hline Factor 2. Síntomas en el estilo de vida & .645 \\
\hline $\begin{array}{l}\text { Síntomas económicos (e.g. pérdida de ingresos, del trabajo o carrera, gastos en seguridad o acciones legales, pérdi- } \\
\text { da de tiempo dedicado a resolver la situación, etc.) }\end{array}$ & .847 \\
\hline $\begin{array}{l}\text { Síntomas conductuales (e.g. tener que cambiar la rutina diaria, cambiar de trabajo, cambiar de horario, cambiar los } \\
\text { hábitos de ejercicio o de ir al gimnasio, cambiar de iglesia, cambiar los hábitos alimenticios, cambiar de pasatiem- } \\
\text { pos, etc.) }\end{array}$ & .672 \\
\hline $\begin{array}{l}\text { Síntomas autodestructivos (e.g. pensamientos acerca de terminar con la vida, hacer planes para terminar con ella, } \\
\text { o intentos con terminar con la vida, etc.) }\end{array}$ & .602 \\
\hline
\end{tabular}


En la Escala de Resiliencia se obtuvo un índice KMO de .828 y la Prueba de Esfericidad de Bartlett de $1957.006(\mathrm{gl}=6 ; p=.000)$ y un solo factor con valor propio superior a 1 que explicó $78.71 \%$ de la varianza (Tabla 2).

Tabla 2. Análisis factorial de la Escala de Resiliencia por reactivo.

\begin{tabular}{|l|c|}
\hline \multicolumn{1}{|c|}{ FACTORES } & $\begin{array}{c}\text { Carga } \\
\text { factorial }\end{array}$ \\
\hline Resiliencia 0 & $\boldsymbol{\alpha}=.911$ \\
\hline $\begin{array}{l}\text { Resiliencia ante la apertura (e.g. sensación de que todo es posible; experimentar una creencia de que se pueden } \\
\text { seguir diferentes caminos en la vida; sentirse capaz de adaptarse a lo que ocurra de ahora en adelante y que todo va } \\
\text { a salir mejor) }\end{array}$ & .926 \\
\hline $\begin{array}{l}\text { Resiliencia de afrontamiento (e.g. sentirse más confiado en su capacidad para manejar este tipo de problemas en el } \\
\text { futuro; darse cuenta de que se es más fuerte de lo que se pensaba; tener una confianza renovada en su habilidad para } \\
\text { seguir adelante y ser mejor en el futuro) }\end{array}$ & .889 \\
\hline $\begin{array}{l}\text { Resiliencia espiritual (e.g. sensación de una mayor apreciación de la importancia de la vida, sensación de optimismo } \\
\text { sobre el futuro, etc.) }\end{array}$ & .883 \\
\hline $\begin{array}{l}\text { Resiliencia social (e.g. sentimiento de renovación en cuanto a lo bueno que son sus relaciones sociales; cuánto } \\
\text { puede depender de su familia, amigos o compañeros de trabajo; tener una actitud más responsable hacia los amigos, } \\
\text { familia o sus propios hijos) }\end{array}$ & .849 \\
\hline M=3.13 D.E. = $\mathbf{1 . 5 2}$ & .89 \\
\hline
\end{tabular}

Para cumplir con el segundo objetivo, primeramente se llevó a cabo una prueba $t$ de Student para muestras independientes para comparar a hombres y las mujeres en cuanto a los síntomas y la resiliencia de manera individualizada, con el propósito de no perder efectos si se comparaban los factores obtenidos en los análisis previos. Los hallazgos muestran diferencias estadísticamente significativas en los síntomas emocionales (e.g. llorar fácilmente, sentirse triste), de ansiedad (e.g. tener ata- ques de pánico, tensión) y mentales (e.g. dificultad para recordar cosas, pensar cosas desagradables) que evidencian que son las mujeres quienes sufren más tales síntomas. En cuanto a la resiliencia, solo se observó una diferencia estadísticamente significativa en la resiliencia espiritual (e.g. sentir una fuerte apreciación de la importancia de la vida), donde también son ellas quienes puntuaron más alto. En los demás reactivos se observaron semejanzas entre los sexos (Tabla 3 ).

Tabla 3. Diferencias sexuales en síntomas y resiliencia.

\begin{tabular}{|l|c|c|c|c|}
\hline \multirow{2}{*}{ Síntomas y resiliencia } & $\begin{array}{c}\text { Mujeres } \\
(\mathbf{n = 5 0 4 )}\end{array}$ & $\begin{array}{c}\text { Hombres } \\
(\mathbf{n = 2 1 3 )}\end{array}$ & \multirow{2}{*}{$\mathbf{t}$} & \multirow{2}{*}{$\boldsymbol{p}$} \\
\cline { 2 - 4 } & $\mathbf{M}$ (D.E.) & M (D.E.) & & \\
\hline Síntomas emocionales & $2.61(1.66)$ & $2.15(1.43)$ & 3.51 & .000 \\
\hline Síntomas de ansiedad & $2.66(1.62)$ & $2.27(1.48)$ & 2.97 & .003 \\
\hline Síntomas mentales & $2.28(1.55)$ & $2.00(1.33)$ & 2.24 & .025 \\
\hline Síntomas sociales & $2.30(1.53)$ & $2.19(1.50)$ & - & - \\
\hline Síntomas espirituales & $1.89(1.41)$ & $1.82(1.37)$ & - & - \\
\hline Síntomas conductuales & $2.10(1.33)$ & $2.08(1.51)$ & - & - \\
\hline Síntomas económicos & $1.58(1.15)$ & $1.70(1.31)$ & - & - \\
\hline Síntomas autodestructivos & $1.49(1.61)$ & $1.42(1.00)$ & - & - \\
\hline Resiliencia ante la apertura & $3.28(1.72)$ & $3.04(1.75)$ & - & - \\
\hline Resiliencia de afrontamiento & $3.48(1.73)$ & $3.45(1.74)$ & - & - \\
\hline Resiliencia espiritual & $2.98(1.77)$ & $2.69(1.71)$ & 2.01 & .044 \\
\hline Resiliencia social & $3.02(1.65)$ & $2.84(1.63)$ & - & - \\
\hline
\end{tabular}


En cuanto a la búsqueda de diferencias en síntomas y resiliencia por tipo de relación con el acosador, se encontró -luego de realizar un análisis de varianza de una vía- que hubo diferencias estadísticamente significativas en los síntomas económicos (e.g. pérdida de ingresos o trabajo, gasto de dinero en seguridad), mentales, emocionales y de ansiedad, siendo mayores cuando el perpetrador del acoso fue una ex pareja o cónyuge, y menores cuando se trató de un extraño o un conocido (Tabla 4).

Tabla 4. Diferencias por tipo de relación con el acosador en síntomas económicos.

\begin{tabular}{|l|c|c|c|c|}
\hline Síntomas económicos & $\begin{array}{c}\text { Económicos } \\
\text { M (D.E.) }\end{array}$ & $\begin{array}{c}\text { Mentales } \\
\text { M (D.E.) }\end{array}$ & $\begin{array}{c}\text { Emocionales } \\
\text { M (D.E.) }\end{array}$ & $\begin{array}{c}\text { Ansiedad } \\
\text { M (D.E.) }\end{array}$ \\
\hline Extraño $(\mathrm{n}=129)$ & $1.44(1.06)$ & $1.95(1.33)$ & $2.19(1.45)$ & $2.30(1.51)$ \\
\hline Conocido $(\mathrm{n}=158)$ & $1.49(1.15)$ & $1.89(1.34)$ & $2.13(1.42)$ & $2.42(1.46)$ \\
\hline Colega $(\mathrm{n}=129)$ & $1.79(1.24)$ & $2.28(1.52)$ & $2.47(1.56)$ & $2.63(1.68)$ \\
\hline Amistad $(\mathrm{n}=95)$ & $1.55(1.22)$ & $2.27(1.49)$ & $2.54(1.77)$ & $2.54(1.64)$ \\
\hline Familiar $(\mathrm{n}=24)$ & $1.71(1.26)$ & $2.13(1.42)$ & $2.88(1.62)$ & $3.04(1.71)$ \\
\hline Cita casual $(\mathrm{n}=34)$ & $1.82(1.21)$ & $2.09(1.28)$ & $2.21(1.17)$ & $2.18(1.04)$ \\
\hline Cita seria $(\mathrm{n}=38)$ & $1.32(0.96)$ & $2.39(1.56)$ & $2.61(1.70)$ & $2.32(1.52)$ \\
\hline Cónyuge $(\mathrm{n}=19)$ & $2.05(1.47)$ & $2.47(1.50)$ & $3.32(1.79)$ & $2.84(1.74)$ \\
\hline Ex pareja(n=53) & $1.96(1.40)$ & $2.94(1.87)$ & $3.45(1.79)$ & $3.12(1.67)$ \\
\hline Otro $(\mathrm{n}=23)$ & $1.52(1.20)$ & $2.83(1.69)$ & $2.91(1.77)$ & $3.17(1.64)$ \\
\hline F $(p)$ & $2 ; 010(036)$ & $3 ; 344(.001)$ & $4 ; 759(.000)$ & $2 ; 283(.016)$ \\
\hline
\end{tabular}

Se hallaron diferencias estadísticamente significativas en dos agrupaciones de reactivos de resiliencia: social (e.g. sentimiento de renovación debido a las relaciones de amigos y familia que se tienen) y afrontamiento (e.g. sentirse con más confianza para manejar este tipo de problemas en el futuro), observándose el mismo efecto que en los síntomas (Tabla 5).

Tabla 5. Diferencias por tipo de relación con el acosador en resiliencia.

\begin{tabular}{|l|c|c|}
\hline \multicolumn{1}{|c|}{ Relación } & $\begin{array}{c}\text { Social } \\
\text { M (D.E.) }\end{array}$ & $\begin{array}{c}\text { Afrontamiento } \\
\text { M (D.E.) }\end{array}$ \\
\hline Extraño $(\mathrm{n}=129)$ & $2.67(1.58)$ & $3.04(1.77)$ \\
\hline Conocido $(\mathrm{n}=158)$ & $2.93(1.68)$ & $3.46(1.76)$ \\
\hline Colega $(\mathrm{n}=129)$ & $3.05(1.63)$ & $3.56(1.61)$ \\
\hline Amistad $(\mathrm{n}=95)$ & $2.70(1.66)$ & $3.40(1.80)$ \\
\hline Familiar $(\mathrm{n}=24)$ & $3.46(1.71)$ & $3.61(1.90)$ \\
\hline Cita Casual $(\mathrm{n}=34)$ & $3.21(1.70)$ & $3.67(1.78)$ \\
\hline Cita Seria $(\mathrm{n}=38)$ & $3.24(1.51)$ & $4.21(1.56)$ \\
\hline Cónyuge $(\mathrm{n}=19)$ & $3.68(1.45)$ & $3.63(1.95)$ \\
\hline Ex pareja $(\mathrm{n}=53)$ & $3.47(1.69)$ & $3.70(1.57)$ \\
\hline Otro $(\mathrm{n}=23)$ & $2.83(1.52)$ & $3.91(1.37)$ \\
\hline F $(p)$ & $2 ; 208 ; .020$ & $2 ; 103 ; .027$ \\
\hline
\end{tabular}

\section{DISCUSIÓN}

Se logró validar en una muestra mexicana las dos escalas de Spitzberg y Cupach (2014) dirigidas a medir los síntomas y la resiliencia en personas víctimas de acoso, lo cual representa una contribución metodológica de la presente investigación. De igual manera, se comprobó que la experiencia del acoso tiene repercusiones graves en la salud de sus víctimas, pero también fortalece sus capacidades resilientes (Spitzberg y Cupach, 2014). Cabe señalar que las opciones de respuesta fueron seleccionadas libremente por los participantes, lo que muestra que la prueba en general es sensible y representa los diferentes niveles de frecuencia con que se manifiestan las variables.

La propuesta original de Spitzberg y $\mathrm{Cu}-$ pach (2014) reporta las escalas como unidimensionales, aunque no se tienen datos de análisis factoriales o confiabilidades (comunicación personal con B. Spitzberg, 6 de marzo de 2013). Sin embargo, al realizar este tipo de análisis en México se encontró que la Escala de Síntomas ante el Acoso mostró un coeficiente de confiabilidad alfa de Conbach total robusto, además de arrojar 
dos factores. El primer factor corresponde a síntomas psicológicos, que contiene reactivos acerca de signos emocionales tales como llorar fácilmente, sentirse deprimido, haber perdido el interés en el sexo, sentir impotencia y soledad; síntomas de ansiedad, como tener ataques de pánico, tensión, paranoia, pesadillas y desconfianza de los demás; síntomas mentales, como dificultad para concentrarse, tomar decisiones, comprender lo que ocurre alrededor o ser obsesivo de las cosas; síntomas sociales, como sentirse menos que los demás, tener arrebatos de ira injustificada o evitar situaciones sociales, y signos espirituales, como perder la fe en todo y todos. El segundo factor, que corresponde a síntomas en el estilo de vida incluye reactivos sobre las implicaciones del acoso en la vida cotidiana; síntomas económicos, como pérdida de ingresos, de trabajo y de dinero gastado en seguridad; síntomas conductuales, como cambiar la rutina y actividades personales y sociales por el acoso, y síntomas autodestructivos, que se orientan hacia la experiencia de pensamientos suicidas (Blaauw, Winkel, Arensman, Sheridan y Freeve, 2002).

Si bien estos factores distan de la propuesta de Spitzberg y Cupach (2014), permiten identificar matices más finos de la experiencia de signos y síntomas ante la IRO en la muestra mexicana. Ambos factores distinguen muy bien las consecuencias negativas, que abarcan no solo lo psicológico (síntomas emocionales, de ansiedad, mentales o cognoscitivos, sociales y espirituales), sino también aquellos efectos en las finanzas o economía de la víctima, sus síntomas evidentes en sus rutinas e intentos de autodestrucción. Al respecto, se ha encontrado que la IRO es una manifestación de estrés postraumático (Nguyen, Spitzberg y Lee, 2012) que puede derivar, según autores como Spitzberg y Cupach (2014) y Tjaden y Thoennes (2000), en enfermedades crónicas, o bien deteriorar en forma importante la salud.

La Escala de Resiliencia -al igual que la reportada por los autores- resultó ser unidimensional y comprende la capacidad de la víctima del acoso para recuperarse y hacer frente a la adversidad mediante la apertura (sensación de que todo es posible y que saldrá bien; el afrontamiento, que favorece la confianza en la capacidad para manejar este tipo de problemas en el futuro; la fortaleza espiritual, por la que la persona siente optimismo ante la vida, y el apoyo social, que involucra un sentimiento de renovación gracias al apoyo de las personas cercanas. Así, la percepción positiva de la red social, las competencias para enfrentar y sobreponerse a la experiencia, la sensación de apreciar la vida y la visión optimista del futuro coinciden con los diferentes acercamientos al constructo de resiliencia (cf. Seligman y Csikszentmihalyi, 2000).

Cuando se compararon los reactivos de síntomas y resiliencia (para reconocer efectos más específicos) entre hombres y mujeres, se observaron grandes similitudes entre ambos, que indican que las víctimas sufren igualmente consecuencias sociales, espirituales, conductuales, económicas y autodestructivos; en cuanto a las diferencias, las mujeres fueron más sintomáticas en lo psicológico, es decir, mostraron prevalencia de problemas emocionales y de memoria y concentración.

Estos resultados reafirman que las mujeres expresan más emociones positivas y negativas y que los hombres procuran ser más racionales ( $\mathrm{Si}$ mon y Nath, 2004); asimismo, se ha encontrado consistentemente en la literatura sobre la IRO que las mujeres manifiestan los síntomas más severos mediante respuestas de ansiedad exacerbada y ataques de pánico (Bjerregaard, 2000; Breiding et al., 2014; Spitzberg y Cupach, 2001, 2014), y que se asustan con mayor facilidad, experimentan más estados de paranoia y modifican más algunos aspectos de su personalidad a raíz del acoso (Davis et al., 2002; Pérez y Sánchez, 2015).

En el caso de la resiliencia, se notaron semejanzas en cuanto a la apertura, el afrontamiento y lo social, así como diferencias en cuanto al reactivo espiritual, en donde también son las mujeres quienes valoran más la vida, lo que podría explicarse en virtud de poseen una sensibilidad mayor que los hombres (Spitzberg et al., 2010), pero también que enfrentan de manera más decidida los sentimientos de vulnerabilidad y desequilibrio emocional resultantes del acoso (Duntley y Buss, 2012).

Finalmente, se examinaron las consecuencias percibidas de tener una relación con el acosador en la sintomatología y el ejercicio de la resiliencia percibidas por la víctima. En el primer caso, se observaron diferencias estadísticamente significativas en algunos de los reactivos (síntomas económicos, mentales, emocionales y de ansiedad), 
que indican que tales síntomas se expresan más frecuentemente cuando la relación es con el cónyuge o la ex pareja, en comparación a cuando el perpetrador es un extraño o un simple conocido. Este efecto quizá se explique mediante algunos hallazgos anteriores, que han señalado que las conductas de acoso están vinculadas a la proximidad que el perpetrador tiene con la víctima, lo que sitúa a los extraños y meros conocidos como acosadores de bajo riesgo (McEwan, Mullen, McKenzie y Ogloff, 2009; Sheridan y Lyndon, 2012). También se ha visto que la forma más frecuente y peligrosa de acoso se produce entre las ex parejas íntimas debido a que tienen mucho más información acerca de la víctima, así como el acceso a sus redes de apoyo, por lo que dirige las conductas de acoso a los aspectos más vulnerables de la vida de aquélla (McEwan, Mullen y McKenzie, 2009; Spitzberg y Cupach, 2007).

En cuanto a la resiliencia, se manifestaron diferencias en el reactivo social y de enfrentamiento, lo que indica que cuando el acosador es el cónyuge o la ex pareja -a diferencia de cuando es un extraño o un amigo-, las relaciones sociales con la familia, los amigos o los compañeros de trabajo representan un gran apoyo que le permite a la víctima sentir más confianza para manejar las situaciones presentes y futuras. Lo anterior se relaciona con las características propias de la muestra del presente estudio, conformada en su mayor número por mujeres, quienes ante una situación de acoso manifestaron ser más propensas a compartir su experiencia con sus amigos, en primer lugar, y después con sus parientes (cf. Edwards, Dardis y Gidycz, 2012). Estas redes de apoyo hacen posible que la víctima se muestre abierta para hablar del suceso y proporcionan apoyo emocional, consejos y acciones tangibles de sostén (Latta y Goodman, 2011; Trotter y Allen, 2009), lo que tiene un efecto positivo en la víctima, desplaza las expresiones de enojo o culpa y fomenta las conductas de afrontamiento (Spitzberg y Cupach, 2014).

El aporte de la presente investigación se centra en la validación de dos escalas destinadas a evaluar los efectos negativos (síntomas) y positivos (resiliencia) en situaciones de acoso relacional en una muestra mexicana, lo que supone, por un lado, contar con dos herramientas válidas y confiables en las áreas de investigación y de ejercicio profesional y, por otro lado, dar peso al papel de la cultura en la forma en la que se acuñan las formas de concebir, sentir y actuar en las relaciones personales, ya que el grupo sociocultural define lo que es apropiado a ciertos contextos interpersonales, las formas de cortejo, los alcances de las conductas durante este proceso e incluso la pertinencia limitar los acercamientos indeseables, como el acoso. De igual manera, esta investigación representa una plataforma para profundizar y reconducir el estudio de las respuestas humanas ante los sucesos traumáticos con una visión no únicamente negativa, centrada en los síntomas, sino utilizando la resiliencia con el fin de desarrollar nuevas formas de intervención basadas en modelos que ayuden a conceptuar, investigar, diseñar e intervenir eficazmente.

\section{REFERENCIAS}

Baum, K., Catalano, S., Rand, M. y Rose, K. (2009). Stalking victimization in the United States (NCJ Report 224527). Washington, D.C.: U.S. Department of Justice.

Bjerregaard, B. (2000). An empirical study of stalking victimization. Violence and Victims, 15, 389-406.

Blaauw, E., Winkel, F.W., Arensman, E., Sheridan, L. y Freeve, A. (2002). The toll of stalking: The relationship between features of stalking and psychopathology of victims. Journal of Interpersonal Violence, 17, 50-63.

Bonanno, G.A. (2004). Loss, trauma and human resilience: have we underestimated the human capacity to thrive after extremely aversive events? American Psychologist, 59(1), 20-28.

Bonanno, G.A., Westphal, M. y Mancini, A.D. (2011). Resilience to loss and potential trauma. Annual Review of Clinical Psychology, 7(1), 511-535.

Breiding, J.M., Smith, S.G., Basile, K.C., Walters, M.I., Chen, J. y Merrick, M.T. (2014). Prevalence and characteristics of sexual violence, stalking, and intimate partner violence victimization - National Intimate Partner and Sexual Violence Survey, United States, 2011. Morbidity and Mortality Weekly Report, 63(8), 1-24.

Brewster, M.P. (1999). An exploration of the experiences and needs of former intimate stalking victims: Final report submitted to the National Institute of Justice. West Chester, UK: West Chester University. 
Casique R., I. (2017). Fuentes y datos sobre la violencia contra las mujeres en México. Aprendizajes, dificultades y retos acumulados. Revista Internacional de Estadística y Geografia, 8(1), 6-16.

Cronbach, L.J. y Meehl, P.E. (1955). Construct validity in psychological tests. Psychological Bulletin, 52(4), 281.

Davis, K.E., Coker, A.L. y Sanderson, M. (2002). Physical and mental health effects of being stalked for men and women. Violence and Victims, 17, 429-443.

Duntley, J.D. y Buss, D.M. (2012). The evolution of stalking. Sex Roles, 66(5-6), 311-327.

Edwards, K., Dardis, C. y Gidycz, C.A. (2012). Women's disclosure of dating violence: A mixed methodological study. Feminism \& Psychology, 22, 507-517. doi: 10.1177/0959353511422280.

Folkman, S. (2013). Stress: appraisal and coping. En M. D. Gellman y J. R. Turner (Eds.): Encyclopedia of Behavioral Medicine (pp. 1913-1915). New York: Springer.

Kamir, O. (2001). Every breath you take: Stalking narratives and the law. Ann Arbor, MI: University of Michigan Press.

Kamphuis, J.H., Emmelkamp, P.M.G. y Bartak, A. (2003). Individual differences in post-traumatic stress following postintimate stalking: Stalking severity and psychosocial variables. British Journal of Clinical Psychology, 42, 145-156.

Kerlinger, F.N. y Lee, H.B. (2002). Investigación del comportamiento. México: McGraw-Hill.

Kuehner, C., Gass, P. y Dressing, H. (2007). Increased risk of mental disorders among lifetime victims of stalking: Findings from a community study. European Psychiatry, 22, 142-145.

Langhinrichsen-Rohling, J. (2012). Gender and stalking: Current intersections and future directions. Sex Roles, 66(5-6), 418-426.

Latta, R.E. y Goodman, L.A. (2011). Intervening in partner violence against women: A grounded theory exploration of informal network members' experiences. The Counseling Psychologist, 39, 973-1023. doi: 10.1177/0011000011398504.

McEwan, T.E., Mullen, P.E. y MacKenzie, R.D. (2009). A study of the predictors of persistence in stalking situations. Law and Human Behavior, 33, 149-158. doi: 10.1007/s10979-008-9141-0.

McEwan, T.E., Mullen, P.E., MacKenzie, R.D. y Ogloff, J.R. (2009). Violence in stalking situations. Psychological Medicine, 39, 1469-1478. doi: 10.1017/S0033291708004996.

McEwan, T.E., Pathé, M. y Ogloff, J.R. (2011). Advances in stalking risk assessment. Behavioral Sciences \& The Law, 29(2), 180-201.

Meloy, J.R. (1999). Stalking: An old behavior, a new crime. Psychiatric Clinics of North America, 22(1), 85-99.

Meloy, J.R., Hoffmann, J., Guldimann, A. y James, D. (2012). The role of warning behaviors in threat assessment: An exploration and suggested typology. Behavioral Sciences \& The Law, 30(3), 256-279.

Miller, L. (2012). Stalking: Patterns, motives, and intervention strategies. Aggression and Violent Behavior, 17(6), 495-506.

Mohandie, K., Meloy, J.R., McGowan, M.G. y Williams, J. (2006). The RECON typology of stalking: Reliability and validity based upon a large sample of North American stalkers. Journal of Forensic Sciences, 51, 147-155.

Mullen, P.E., Pathé, M. y Purcell, R. (2001). Stalking: new constructions of human behaviour. Australian and New Zealand Journal of Psychiatry, 35(1), 9-16.

Nguyen, L.K., Spitzberg, B.H. y Lee, C.M. (2012). Coping with obsessive relational intrusion and stalking: The role of social support and coping strategies. Violence and Victims, 27(3), 414-433.

Nicastro, A.M., Cousins, A.V. y Spitzberg, B.H. (2000). The tactical face of stalking. Journal of Criminal Justice, 28(1), 69-82.

Ozer, E.J., Best, S.R., Lipsey, T.L. y Weiss, D.S. (2003). Predictors of posttraumatic stress disorder and symptoms in adults: a meta-analysis. Psychological Bulletin, 129(1), 52-73.

Pérez P., A.E. y Sánchez A., R. (2015). Efectos positivos y negativos del contacto mediado en mujeres víctimas de acoso. Revista Iberoamericana de Psicología: Ciencia y Tecnología, 8(2), 99-109.

Reyes L., I. y García B., L.F. (2008). Hacia un procedimiento de validación psicométrica culturalmente relevante. En S. Rivera A., R. Díaz-Loving, R. Sánchez A. e I. Reyes L (Eds.): La Psicología Social en México (pp. 625-630). México: AMEPSO.

Seligman, M.E. y Csikszentmihalyi, M. (2000). Positive psychology: An introduction. American Psychologist, January, 55(1), $5-14$.

Sheridan, L. y Lyndon, A.E. (2012). The influence of prior relationship, gender, and fear on the consequences of stalking victimization. Sex Roles, 66(5-6), 340-350.

Simon, R.W. y Nath, L.E. (2004). Gender and emotion in the United States: Do men and women differ in self-reports of feelings and expressive behavior? American Journal of Sociology, 109(5), 1137-1176.

Sinclair, H.C. (2012). Stalking myth-attributions: Examining the role of individual and contextual variables on attributions in unwanted pursuit scenarios. Sex Roles, 66(5), 378-391. doi: 10.1007/s11199-010-9853-8.

Spitzberg, B.H. y Cupach, W.R. (2001). Paradoxes of pursuit: Toward a relational model of stalking-related phenomena. En J.A. Davies (Ed.): Stalking crimes and victim protection. Prevention, intervention, threat assessment, and case management (pp. 97-136). Boca Raton, FL: CRC Press. 
Spitzberg, B.H. y Cupach, W.R. (2007). The state of the art of stalking: Taking stock of the emerging literature. Aggression and Violent Behavior, 12, 64-86.

Spitzberg, B.H. y Cupach, W.R. (2014). The dark side of relationship pursuit: From attraction to obsession and stalking. New York: Routledge.

Spitzberg, B.H., Cupach, W.R. y Ciceraro, L.D. (2010). Sex differences in stalking and obsessive relational intrusion: Two meta-analyses. Partner Abuse, 1(3), 259-285.

Tjaden, P. y Thoennes, N. (2000). Prevalence and consequences of male-to-female and female-to-male intimate partner violence as measured by the national violence against women survey. Violence Against Women, 6, 142-161. doi: 10.1177/ 10778010022181769.

Trotter, J.L. y Allen, N.E. (2009). The good, the bad, and the ugly: Domestic violence survivors' experiences with their informal sources. American Journal of Community Psychology, 43, 221-231. doi: 10.1007/s10464-009-9232-1.

Tugade, M.M. y Fredrickson, B.L. (2004). Resilient individuals use positive emotions to bounce back from negative emotional experiences. Journal of Personality and Social Psychology, 86(2), 320-333. 